\title{
Back on Stochastic Model for Sandpile*
}

\author{
Noureddine IGBIDA ${ }^{\dagger}$
}

June 5, 2009

\begin{abstract}
Our aim in this note is to give a simplified proof of the convergence of Evans-Rezakhanlou Stochastic Model to the evolution surfaces model of sandpile.
\end{abstract}

\section{Introduction}

The stochastic model for sandpile was introduced by Evans and Rezakhanlou in [6] as a variant of physical models for sandpile. It corresponds to a Markov process $(X(t))_{t \geq 0}$ defined by an infinitesimal generator describing the evolution of stack of unit cubes resting on the plane when new cubes are being added to the pile, by being placed either upon a heretofore unoccupied square in the plane or else upon the top of a current column.

\section{Example :}

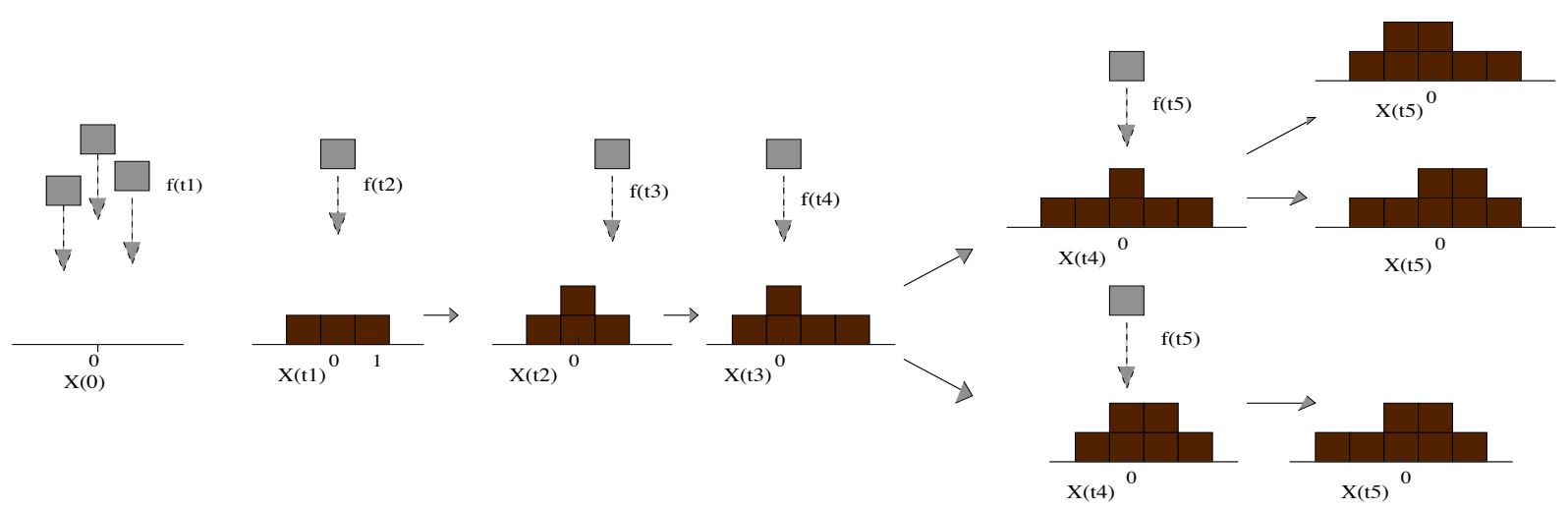

At each time $t>0$, the configuration $X(t)$ needs to be stable which means that the heights of any two adjacent columns of cubes can differ by at most one. So adding new cubes on existing pile, we ordain two possibilities to each cube :

\footnotetext{
*Work supported by the French A.N.R. Grant JC05-41831 and by "FLUPARTI" project (supported and funded by "Conseil Régional de Picardie").

${ }^{\dagger}$ LAMFA, UMR 6140, Université de Picardie Jules Verne, 33 rue Saint Leu, 80038 Amiens, France. Email : noureddine.igbida@u-picardie.fr
} 
- if the configuration is stable, then the cube remains in place

- otherwise, the cube has several downhill "staircases" along which it can move, and the cube will randomly select among the allowable downhill paths.

If we consider the case where the cubes can be also taken away, then a third possibility may be ordain

- the cube has several "upward staircases" along which it can move, and the cube will randomly select among the allowable upward paths.

Assuming that we continuously add cubes at random locations on a starting empty stack, Evans and Rezakhanlou studies the limit when one rascals in both space and time, so as to consider growing piles of more and more smaller and smaller cubes? They prove that the macroscopic limit is rather simple and very connected Prigozhin model for sandpile (cf. [8] and [2]).

Since the work of Prigohzin (cf. [8] see also [2]), this has been well known (see also [3], [6] and the references therein) that the evolution of the surface of the sandpile when the angle of stability is equal to $\pi / 4$ can be described by the following evolution problem

$$
\left\{\begin{array}{l}
\partial_{t} u+\partial I_{K}(u) \ni f \\
\hat{u}(0)=0
\end{array}\right.
$$

where $K=\left\{z \in W^{1, \infty}\left(\mathbb{R}^{2}\right) \cap L^{2}\left(\mathbb{R}^{2}\right) ;|\nabla z| \leq 1\right\}, \partial \mathbb{I}_{K}$ denotes the sub-differential operator of the indicator function (cf. [4]) of $K$ and $f$ describes a source term . Solution $u$ is the height of the surface that grows up (resp. grows down) under sand addition (resp. sand removal) by a source called $f$. This is a critical slope model obtained by using the continuity equation and the gradient constraint $|\nabla u| \leq 1$ a.e. in $\mathbb{R}^{2}$ (see [8] and [2] for more details). Existence, uniqueness and numerical approximation the solution are well known by now for this model (cf. [8], [2], [5] and [7]). More precisely, for any $f \in B V_{l o c}\left(0, T ; L^{2}\left(\mathbb{R}^{2}\right)\right)$, we know that that (1) has a unique solution $u$ in the sense that $u \in W_{l o c}^{1, \infty}\left(0, \infty ; L^{2}\left(\mathbb{R}^{2}\right)\right), u(0)=0$ and, for any $t \geq 0, u(., t) \in K$ and

$$
\int_{\mathbb{R}^{2}}\left(f(t, x)-\partial_{t} u(t, x)\right)(u(t, x)-\xi(x)) \geq 0 \quad \text { for any } \xi \in K .
$$

To give the connection between the two models, let $N$ be a large integer. Assume the cubes are of side length $O\left(N^{-1}\right)$ and cubes newly and randomly added at rate $O\left(N^{-1}\right)$ are continuously falling downhill. For the description of the evolving of cubes, the authors of [6] introduce a probabilistic lattice model. They thus consider a Markov process for the height $X(t, i)$, defined for times $t \geq 0$ and sites $i \in \mathbb{Z}^{2}$. Resacaled source terms $f^{+}\left(\frac{t}{N}, \frac{i}{N}\right)$ and $f^{-}\left(\frac{t}{N}, \frac{i}{N}\right)$ control the rate new cubes are added to the pile or removed from it. Then, they proved that

$$
\mathbb{E}\left[\int_{\mathbb{R}^{2}}\left|\frac{1}{N} X(N t,[N x])-u(t, x)\right|^{2}\right] \rightarrow 0 \quad \text { as } N \rightarrow \infty
$$


where $u$ is the unique solution of (1). As shown in [6], the key estimates for the proof (3) are some kind of microscopic version of (2) (see (10)). To prove these estimates the authors of [6] prove some elementary intermediate estimates evolving various types of sets. Our aim in this note, is to improve these key estimates directly by using some simple arguments.

In the next section, we recall the stochastic model of Evans and Rezakhanlou. In Section 3, we prove the key estimates (10). Then, as in [6], we introduce the discrete evolution problem associated with (1). Then, we give the proof of (3) by using some results of [1].

\section{The stochastic model for sandpile problem (cf. [6])}

To describe the stochastic process for sandpile, we consider the lattice $\mathbb{Z}^{n}$. We equipped $\mathbb{Z}^{n}$ with an Euclidean norm and we say that $i, j \in \mathbb{Z}^{n}$ are adjacent, written $i \sim j$, provided

$$
|i-j| \leq 1
$$

Without loose of generality, we restrict ourself to the cases $n=2$, and we write $i=\left(i_{1}, i_{2}\right)$ to denote a typical site in $\mathbb{Z}^{2}$. Then, we introduce the Hilbert space

$$
H:=l^{2}\left(\mathbb{Z}^{2}\right)=\left\{X: \mathbb{Z}^{2} \rightarrow \mathbb{R} ;\|X\|:=\sum_{i} X(i)^{2}<\infty\right\} .
$$

A (stable) configuration is a mapping $X: \mathbb{Z}^{2} \rightarrow \mathbb{Z}$ such that

$$
\left\{\begin{array}{l}
|X(i)-X(j)| \leq 1 \quad \text { if } i \sim j \\
\text { and } X \text { has bounded support. }
\end{array}\right.
$$

The state space is

$$
S:=\left\{X: \mathbb{Z}^{2} \rightarrow \mathbb{Z} ; X \text { is a configuration }\right\},
$$

and the set of stable configuration is

$$
\hat{K}:=\{X \in H ;|X(i)-X(j)| \leq 1 \text { if } i \sim j\} .
$$

Let $\hat{f}:(0, \infty) \times \mathbb{Z}^{2} \rightarrow \mathbb{Z}$ be a function, such that $f^{+}$(resp. $\left.f^{-}\right)$is controlling the rate new cubes are added (resp. removed) to the pile. The source of cubes $\hat{f}$ generates a stochastic process $(X(t), t \geq 0)$ in the state space $S$. It is clear that the probability that $X(t)$ be situated (at time $t$ ) in a given set $\Gamma$ of $E$, under the condition that the movement of the system up to time $s(s<t)$ is completely known, depends only on the state of the system at time $s$. In other words $(X(t), t \geq 0)$ is a Markov process. To study this process, w need to know it's infinitesimal generator $A$, or more precisely $A F(X(t))$ for any $F \in B(S)$, the set of bounded functions. To this aim, let us consider $p^{+}(i, j, \xi)$ the probability that a cube placed on a given configuration $\xi \in S$ at the position $i$ will end up at $j$ after it has fallen downward over the stack with eight 
$\xi$. Likewise, let $p^{-}(i, j, \xi)$ be the probability that the removal of a cube from the pile at $i$ will result in a removal at site $j$, after the cubes along a staircase each shifts downwards to fill in the gap created at site $i$. So, for any $i, j \in \mathbb{Z}^{2}$ we have

$$
0 \leq p^{ \pm}(i, j, \xi) \leq 1 \quad \text { and } \quad \sum_{i \in \mathbb{Z}^{2}} p^{ \pm}(i, j, \xi)=1 .
$$

Thanks to [6], we consider $c^{ \pm}$given by

$$
c^{ \pm}(j, X, t)=\sum_{i \in \mathbb{Z}^{2}} p^{ \pm}(i, j, X(t)) \hat{f}^{ \pm}(t, i), \quad \text { for any }(t, j) \in \mathbb{Z}^{2} \times[0, \infty) .
$$

The parameter $c^{+}(j, X, \tau)$ (resp. $\left.c^{-}(j, X, \tau)\right)$ is highly nonlocal factor and records the rate, at time $\tau$, new cubes come to rest at the site $j$ after falling downhill (resp. the rate at which cubes are removed from the site $j$, at time $\tau$ ). In particular, we have

$$
\sum_{j \in \mathbb{Z}^{2}} c(j, X, t)=\sum_{i \in \mathbb{Z}^{2}} f(t, i)
$$

Thanks again to [6], the infinitesimal generator $A$ of the Markov process $(X(t), t \geq 0)$, is given by

$$
\begin{aligned}
A F(X(t))= & \sum_{j \in \mathbb{Z}^{N}} c^{+}(j, X, t)\left(F\left(X(t)+\delta_{j}\right)-F(X(t))\right) \\
& \quad-\sum_{j \in \mathbb{Z}^{N}} c^{-}(j, X, t)\left(F\left(X(t)-\delta_{j}\right)-F(X(t))\right) \quad \text { for any } t \geq 0,
\end{aligned}
$$

where, for any $j \in \mathbb{Z}^{2}, \delta_{j}: \mathbb{Z}^{2} \rightarrow \mathbb{N}$ is given by

$$
\delta_{j}(i)= \begin{cases}1 & \text { if } i=j \\ 0 & \text { otherwise }\end{cases}
$$

In particular, we have

$$
A F(X(t))=\left(\mathcal{L}_{t} F\right)(X(t)) \quad \text { for any }(t, F) \in(0, \infty) \times B(S),
$$

where $\mathcal{L}_{t}: F \in B(S) \rightarrow \mathcal{L}_{t} F \in L(S, \mathbb{R})$, for any $t \geq 0$, is the time dependent operator defined by

$$
\left(\mathcal{L}_{t} F\right)(\xi):=\sum_{j \in \mathbb{Z}^{N}} c^{+}(j, \xi, t)\left(F\left(\xi+\delta_{j}\right)-F(\xi)\right)-\sum_{j \in \mathbb{Z}^{N}} c^{-}(j, \xi, t)\left(F\left(\xi-\delta_{j}\right)-F(\xi)\right) .
$$




\section{Main results}

Thanks to [6], we know that the connection between the stochastic model and (1) is given through the following nonlinear dynamic in $l^{2}\left(\mathbb{Z}^{2}\right)$ :

$$
\left\{\begin{array}{l}
\partial_{t} \hat{u}+I_{\hat{K}}(\hat{u}) \ni \hat{f} \quad \text { for } t \geq 0 \\
\hat{u}(0)=0
\end{array}\right.
$$

where $\partial I_{\hat{K}}$ denotes the sub-differential of $I_{\hat{K}}$ in $l^{2}\left(\mathbb{Z}^{2}\right)$. Since $\hat{K}$ is closed and convex with $S \subseteq \hat{K}$, then (cf. [4]) for a given $\hat{f} \in B V\left(0, T ; l^{2}\left(\mathbb{Z}^{2}\right)\right.$ ), the evolution (8) has a unique solution $\hat{u} \in W_{l o c}^{1, \infty}(0, \infty ; H)$ such that $\hat{u}(0)=0$ and, for any $t \geq 0, \hat{u}(., t) \in \hat{K}$ and

$$
\sum_{i}\left(\hat{f}(t, i)-\partial_{t} \hat{u}(t, i)\right)(\hat{u}(t, i)-\hat{\xi}(i)) \geq 0 \quad \text { for any } \hat{\xi} \in \hat{K} .
$$

We have

Theorem 1 Assume that $\hat{f} \in B V\left(0, T ; l^{2}\left(\mathbb{Z}^{2}\right)\right.$. Let $\hat{u}$ be the solution of $(8)$ and $(X(t), t \geq 0)$ be the stochastic process generated by $\hat{f}$. Then, we have

$$
\left.\mathbb{E}\left[\sum_{i \in \mathbb{Z}^{2}}(X(t, i)-\hat{u}(t, i))^{2}\right] \leq \int_{0}^{t} \sum_{j \in \mathbb{Z}^{2}} \hat{f}(j, s)\right) d s, \quad \text { for any } t \geq 0 .
$$

Thanks to [6], recall that the proof of Theorem 1 is based on the following estimates.

Lemma 1 Under the assumptions of Theorem 1, for any $w \in S$, we have

$$
\pm \sum_{j \in \mathbb{Z}^{2}} c^{ \pm}(j, X, t)(X(t, j)-w(j)) \leq \pm \sum_{i \in \mathbb{Z}^{2}} \hat{f}(t, i)(X(t, i)-w(i)) \quad \forall w \in S
$$

The estimates (10) are some kind of microscopic version of (2). To prove (10) the authors of [6] prove some elementary intermediate estimates evolving various types of sets. In these notes, we use essentially the following remark and give a direct and short proof of this result. Then, the proof of Theorem 1 follows more or less the same step of [6]. Indeed, Lemma 1 gives the connection between $X(t)$ and (8). Then, we transform (8) into an evolution problem in $L^{2}\left(\mathbb{R}^{2}\right)$ governed by a sub-differential operator of the indicator function of the set of rescaled configurations (see (14)). This transformation allows us to give the connection between (1), (15) and the stochastic model. 
Remarks 1. For a given $\xi \in S$, if $p^{+}(i, j, \xi)>0$, then there exists at least one staircase $i_{0}=i \sim i_{1} \sim \ldots \sim i_{m}=j$, such that $\xi\left(i_{p}\right)=\xi\left(i_{p+1}\right)+1$ for any $p=0,1, \ldots m-1$. Let us denote this staircase by $\mathcal{C}^{+}(i, j)$; i.e.

$$
\mathcal{C}^{+}(i, j)=\left[i_{0}, i_{1}, \ldots, i_{m-1}, j\right],
$$

and, for any $k \in \mathcal{C}^{+}(i, j)$, we denote by $\tilde{k}$, the adjacent side to $k$ such that $u(k)=u(\tilde{k})+1$. It is clear that $\mathcal{C}(i, j)$ may not be unique, so (it is not essential) to simplify the presentation, let us consider the application :

$$
\mathcal{C}^{+}: \mathbb{Z}^{2} \times \mathbb{Z}^{2} \rightarrow \mathcal{C}^{+}(i, j) \text { a staircase between } i \text { and } j \text {. }
$$

2. For a given $\xi \in S$, if $p^{-}(i, j, \xi)>0$, then there exists at least one upward staircase $i_{0}=$ $i \sim i_{1} \sim \ldots \sim i_{m}=j$, such that $\xi\left(i_{p}\right)=\xi\left(i_{p+1}\right)+1$ for any $p=0,1, \ldots m-1$. Let us denote this staircase by $\mathcal{C}^{-}(i, j)$; i.e.

$$
\mathcal{C}^{-}(i, j)=\left[i_{0}, i_{1}, \ldots, i_{m-1}, j\right]
$$

and, for any $k \in \mathcal{C}(i, j)$, we denote by $\tilde{k}$, the adjacent side to $k$ such that $u(k)=u(\tilde{k})-1$. It is clear that $\mathcal{C}(i, j)$ may not be unique, so (it is not essential) to simplify the presentation, let us consider the application :

$$
\mathcal{C}^{-}: \mathbb{Z}^{2} \times \mathbb{Z}^{2} \rightarrow \mathcal{C}^{-}(i, j) \text { a staircase between } i \text { and } j .
$$

Proof of Lemma 1: Thanks to (4), we have

$$
\begin{aligned}
\sum_{j \in \mathbb{Z}^{2}} c^{+}(j, X, t)(X(t, j)-w(j))= & \sum_{j, i \in \mathbb{Z}^{2}} p^{+}(i, j, X(t)) \hat{f}^{+}(t, i)(X(t, j)-w(j)) \\
= & \sum_{j, i \in \mathbb{Z}^{2}} p^{+}(i, j, X(t)) \hat{f}^{+}(t, i)(X(t, i)-w(i)) \\
& +\sum_{j, i \in \mathbb{Z}^{2}} p^{+}(i, j, X(t)) \hat{f}^{+}(t, i)((w(i)-w(j))-(X(t, i)-X(t, j))) \\
= & I_{1}+I_{2}
\end{aligned}
$$

Since $\sum_{j \in \mathbb{Z}^{2}} p^{+}(i, j, X(t))=1$, for any $(t, i) \in \mathbb{Z}^{2} \times(0, \infty)$, then it is clear that

$$
I_{1}=\sum_{i \in \mathbb{Z}^{2}} \hat{f}^{+}(t, i)(X(t, i)-w(i))
$$


Let us prove that $I_{2} \leq 0$. Thanks to Remark, we have

$$
\begin{aligned}
I_{2} & =\sum_{j, i \in \mathbb{Z}^{2}} p^{+}(i, j, X(t)) \hat{f}^{+}(t, i) \sum_{k \in \mathcal{C}^{+}(i, j)}((w(k)-w(\tilde{k}))-(X(t, k)-X(\tilde{k}, t))) \\
& \leq \sum_{j, i \in \mathbb{Z}^{2}} p^{+}(i, j, X(t)) \hat{f}^{+}(t, i) \sum_{k \in \mathcal{C}^{+}(i, j)}((w(k)-w(\tilde{k}))-1) \\
& \leq 0,
\end{aligned}
$$

where we used the fact that $|w(k)-w(\tilde{k})| \leq 1$ (since $w \in K$ and $k \sim \tilde{k}$ ). The proof of

$$
\sum_{j \in \mathbb{Z}^{2}} c^{-}(j, X, t)(X(t, j)-w(j)) \geq \sum_{i \in \mathbb{Z}^{2}} \hat{f}^{-}(t, i)(X(t, i)-w(i)),
$$

follows in the same way, we let the details to the reader.

To simplify the presentation of the proof of Theorem 1, we divide the proof into two steps that we present in the following Lemmas.

Lemma 2 Under the assumptions of Theorem 1, we have

1. For any $w \in S$ and $t \geq 0$, we have

$$
\frac{1}{2} \mathcal{L}_{t}\left(\sum_{i \in \mathbb{Z}^{2}}(X(t, i)-w(i))^{2}\right) \leq \sum_{j \in \mathbb{Z}^{2}} \hat{f}(t, j)(X(t, j)-w(j))+\frac{1}{2} \sum_{j \in \mathbb{Z}^{2}} f(t, j) .
$$

2. For any $w \in W^{1, \infty}(0, T ; H)$ such that $w(t) \in S$, for any $t \in[0, T)$, we have

$$
\begin{aligned}
\frac{1}{2} \sum_{i \in \mathbb{Z}^{2}}(X(t, i)-w(t, i))^{2} \leq & \int_{0}^{t}\left[\sum_{i \in \mathbb{Z}^{2}} \frac{\partial w}{\partial s}(i, s)(w(i, s)-X(i, s))\right. \\
& \left.+\sum_{j \in \mathbb{Z}^{2}} f(j, s)(X(j, s)-w(j, s))+\frac{1}{2} \sum_{j \in \mathbb{Z}^{2}} f(j, s)\right]+\mathcal{M}(t)
\end{aligned}
$$

where $(\mathcal{M}(t))_{t \geq 0}$ is a martingale satisfying

$$
\mathbb{E}(\mathcal{M}(t))=0 \quad \text { for any } t \geq 0 \text {. }
$$

Proof : 
1. Let us denote

$$
I=\frac{1}{2} \mathcal{L}_{t}\left(\sum_{i \in \mathbb{Z}^{2}}(X(t, i)-w(i))^{2}\right)
$$

By definition of $\mathcal{L}$, we have

$$
I=\frac{1}{2} \sum_{j \in \mathbb{Z}^{2}} c(j, X, t)\left(\sum_{i \in \mathbb{Z}^{2}}\left(T_{j}(X(t))(i)-w(i)\right)^{2}-\sum_{i \in \mathbb{Z}^{2}}(X(t, i)-w(i))^{2}\right) .
$$

So,

$$
\begin{aligned}
I & =\frac{1}{2} \sum_{j \in \mathbb{Z}^{2}} c(j, X, t)\left(\sum_{i \in \mathbb{Z}}\left(T_{j}(X)(i)-X(i)\right)\left(T_{j}(X)(i)+X(i)-2 u(i)\right)\right) \\
& =\frac{1}{2} \sum_{j \in \mathbb{Z}^{2}} c(j, X, t)(2 X(j)+1-2 u(j)) \\
& =\sum_{j \in \mathbb{Z}^{2}} c(j, X, t)(X(j)-u(j))+\frac{1}{2} \sum_{j \in \mathbb{Z}^{2}} c(j, X, t) .
\end{aligned}
$$

Thanks to (4), we deduce that

$$
I=\sum_{j \in \mathbb{Z}^{2}} c(j, X, t)(X(j)-u(j))+\frac{1}{2} \sum_{j \in \mathbb{Z}^{2}} f(t, j),
$$

and, using Lemma 1, the first step of the lemma follows.

2. As in [6], we use the following stochastic integral equation : for any $F: S \times(0, \infty) \rightarrow \mathbb{R}$ Lipchitz continuous in $t$ and $F(X(., 0), 0)=0$, we have

$$
F(X(., t), t)=\int_{0}^{t}\left(\frac{\partial F}{\partial s}+\mathcal{L}_{s} F\right)(X(., s))+\mathcal{M}(t) .
$$

Let $F$ be given by

$$
F(\xi, t)=\frac{1}{2} \sum_{i \in \mathbb{Z}^{2}}(\xi(i)-w(t, i))^{2}, \quad \text { for any }(\xi, t) \in S \times(0, T) .
$$

Then,

$$
\frac{\partial F}{\partial s}(\xi, s)=-\sum_{i \in \mathbb{Z}^{2}} \frac{\partial w}{\partial s}(i, s)(\xi(i)-w(i, s)), \quad \text { for any }(\xi, t) \in S \times(0, T),
$$

and (11) implies that, for any $t \geq 0$,

$\frac{1}{2} \sum_{i \in \mathbb{Z}^{2}}(X(t, i)-w(t, i))^{2}=\int_{0}^{t}\left(\sum_{i \in \mathbb{Z}^{2}} \frac{\partial w}{\partial s}(i, s)(w(i, s)-X(i, s))+\mathcal{L}_{s}(F(X(., s), s)+\mathcal{M}(t)\right.$.

Then, by using the first step, the result follows. 
Proof of Theorem 1 : Using the fact that $\hat{u}$ is a solution of $(8)$ and $X(t) \in \hat{K}$, for any $t \geq 0$, we have

$$
\sum_{i \in \mathbb{Z}^{2}} \frac{\partial \hat{u}}{\partial s}(i, s)(\hat{u}(i, s)-X(i, s))+\sum_{j \in \mathbb{Z}^{2}} \hat{f}(j, s)(X(j, s)-\hat{u}(j, s)) \leq 0 \quad \text { for any } t \geq 0 .
$$

Then, using the second part of Lemma 2 with $\hat{u}$, we deduce (9).

At last, let us come back to the continuous model of Prigozhin (1) and assume that

$$
f \in B V_{l o c}\left(0, \infty ; L^{2}\left(\mathbb{R}^{2}\right)\right) \text { and there exists } R>0 \text { such that } \operatorname{spt}(f) \subseteq B(0, R),
$$

here $B(0, R)$ denotes the a ball with center 0 and radius $R$. Thanks to [4], we know that (1) has a unique solution $u$ in the sense that $u \in W_{l o c}^{1, \infty}\left(0, \infty ; L^{2}\left(\mathbb{R}^{2}\right)\right), u(0)=0$ and, for any $t \geq 0$, $u(., t) \in K$ and

$$
\int_{\mathbb{R}^{2}}\left(f(t, x)-\partial_{t} u(t, x)\right)(u(t, x)-\xi(x)) \geq 0 \quad \text { for any } \xi \in K .
$$

For the connection with the stochastic model, we rescale the source term $f\left(\frac{t}{N}, \frac{x}{N}\right)$. We set

$$
\hat{f}(t, i)=f\left(\frac{t}{N}, \frac{x}{N}\right) \quad \text { for any }(t, i) \in[0, \infty) \times \mathbb{Z}^{2},
$$

and we consider $(X(t))_{t \geq 0}$ the Markov processus generated by $\hat{f}$ as explained in the previous section.

Theorem 2 Under the assumption (12), we have

$$
\mathbb{E}\left[\int_{\mathbb{R}^{2}}\left|u(t, x)-\frac{1}{N} X(N t,[N x])\right|^{2}\right] \rightarrow 0 \quad \text { as } N \rightarrow 0
$$

Proof : Let $\hat{u}$ be the solution of (8) and consider

$$
u_{N}(t, x)=\frac{1}{N} \hat{u}(N t,[N x]) \quad \text { and } \quad f_{N}(t, x)=f\left(t, \frac{[N x]}{N}\right) \quad \text { for any }(t, x) \in[0, T) \times \mathbb{R}^{2} .
$$

It is not difficult to see that $u_{N} \in W_{l o c}^{1, \infty}\left((0, \infty) ; L^{2}\left(\mathbb{R}^{2}\right)\right)$ and, for any $t \geq 0, u_{N}(., t) \in K_{N}$, where

$$
K_{N}=\left\{z \in L^{2}\left(\mathbb{R}^{2}\right) ;|u(x)-u(y)| \leq \frac{1}{N} \text { for }|x-y| \leq \frac{1}{N}\right\} .
$$


In addition, for any $\xi \in K_{N}$, we have

$\int_{\mathbb{R}^{2}}\left(f_{N}(t, x)-\partial_{t} u_{N}(t, x)\right)\left(u_{N}(t, x)-\xi(x)\right)=\frac{1}{N^{3}} \sum_{i}\left(\hat{f}(N t, i)-\partial_{t} \hat{u}(N t, i)\right)(\hat{u}(N t, i)-\hat{\xi}(i))$,

where

$$
\hat{\xi}(i)=N^{3} \int_{I_{i}} \xi(x) d x \quad \text { and } \quad I_{i}=\left\{z \in \mathbb{R}^{2} ;[N z]=i\right\} .
$$

Now, since $\xi \in K_{N}$ and $\hat{u}$ is the solution of (8), then $\hat{\xi} \in \hat{K}$ and

$$
\int_{\mathbb{R}^{2}}\left(f_{N}(t, x)-\partial_{t} u_{N}(t, x)\right)\left(u_{N}(t, x)-\xi(x)\right) \geq 0 \text { for any } \xi \in K_{N} .
$$

In other words $u_{N}$ is the solution of the nonlinear dynamic

$$
\left\{\begin{array}{l}
\partial_{t} u_{N}+\partial I_{K_{N}}\left(u_{N}\right) \ni \hat{f}_{N} \\
\hat{u}_{N}(0)=0 .
\end{array}\right.
$$

Thanks to [1], we know that

$$
u_{N} \rightarrow u \quad \text { in } \mathcal{C}\left([0, T) ; L^{2}\left(\mathbb{R}^{2}\right)\right) .
$$

Indeed, $\cap_{N \in \mathbb{N}} K_{N}=K$ so that, letting $N \rightarrow \infty$, we have $\partial I_{K_{N}}$ converges in the sense of graph to $\partial \mathbb{I}_{K}$ in $L^{\infty}\left(\mathbb{R}^{2}\right)$. On the other hand, as $N \rightarrow \infty$,

$$
f_{N} \rightarrow f \quad \text { in } L_{l o c}^{2}\left((0, \infty) ; L^{2}\left(\mathbb{R}^{2}\right)\right) .
$$

Then, by using classical perturbation result for nonlinear semigroup (cf. [4]), (16) follows. At last, thanks to Theorem 1, we have

$$
\begin{aligned}
\mathbb{E}\left[\int_{\mathbb{R}^{2}}\left(\frac{1}{N} X(N t,[N x])-u_{N}(t, x)\right)^{2} d x\right] & =\mathbb{E}\left[\frac{1}{N^{4}} \sum_{i \in \mathbb{Z}^{2}}\left|X(N t, i)-N u_{N}(t, i)\right|^{2} d x\right] \\
& =\mathbb{E}\left[\frac{1}{N^{4}} \sum_{i \in \mathbb{Z}^{2}}|X(N t, i)-\hat{u}(N t, i)|^{2} d x\right] \\
& \leq \frac{1}{N^{4}} \int_{0}^{N t} \sum_{i \in \mathbb{Z}^{2}} \hat{f}(s, i) d s \\
\leq & \frac{1}{N^{2}} \int_{0}^{N t} \int_{\mathbb{R}^{2}} f\left(\frac{s}{N}, \frac{[N x]}{N}\right) d s d x \\
\leq & \frac{1}{N} \int_{0}^{t} \int_{\mathbb{R}} f\left(\tau, \frac{[N x]}{N}\right) d \tau d x \\
& \rightarrow 0 \quad \text { as } N \rightarrow \infty .
\end{aligned}
$$

so, by using (16), we deduce (13). 


\section{References}

[1] F. Andreu, J. M. Mazon, J. D. Rossi and J. Toledo, The limit as $p \rightarrow \infty$ in a nonlocal $p$-Laplacian evolution equation. A nonlocal approximation of a model for sandpiles. To appear in Cal. Var. Partial Diff. Equ.

[2] G. Aronson, L. C. Evans and Y. Wu, Fast/Slow diffusion and growing sandpiles. J. Differential Equations, 131:304-335, 1996.

[3] J. W. Barrett and L. Prigozhin, Dual formulation in Critical State Problems. Interfaces and Free Boundaries, 8, 349-370, 2006.

[4] H. Brézis, Opérateurs maximaux monotones et semigroups de contractions dans les espaces de Hilbert. (French). North-Holland Mathematics Studies, No. 5. Notas de Matemàtica (50). North-Holland Publishing Co., Amsterdam-London; American Elsevier Publishing Co., Inc., New York, 1973.

[5] S. Dumont and N. Igbida, Back on a Dual Formulation for the Growing Sandpile Problem. To appear in Eur. Journal Appl. Math..

[6] L. C. Evans and F. Rezakhanlou. A stochastic model for sandpiles and its continum limit. Comm. Math. Phys., 197 (1998), no. 2, 325-345.

[7] N. Igbida Evolution Monge-Kantorovich equation. submitted.

[8] L. Prigozhin, Variational model of sandpile growth. Euro. J. Appl. Math. , 7, 225-236, 1996 